\title{
Novel high-speed camera analysis of transverse mode instabilities in rod fiber amplifiers
}

Christensen, Simon Lønborg; Johansen, Mette M. ; Michieletto, Mattia ; Triches, Marco ; Hout, Laurent; Maack, Martin D.; Lægsgaard, Jesper

\section{Published in:}

Proceedings of SPIE

Link to article, DOI:

$10.1117 / 12.2542673$

Publication date:

2020

Document Version

Publisher's PDF, also known as Version of record

Link back to DTU Orbit

Citation (APA):

Christensen, S. L., Johansen, M. M., Michieletto, M., Triches, M., Hout, L., Maack, M. D., \& Lægsgaard, J. (2020). Novel high-speed camera analysis of transverse mode instabilities in rod fiber amplifiers. In Proceedings of SPIE (Vol. 11260). [1126017] SPIE - International Society for Optical Engineering. Proceedings of SPIE - The International Society for Optical Engineering https://doi.org/10.1117/12.2542673

\section{General rights}

Copyright and moral rights for the publications made accessible in the public portal are retained by the authors and/or other copyright owners and it is a condition of accessing publications that users recognise and abide by the legal requirements associated with these rights.

- Users may download and print one copy of any publication from the public portal for the purpose of private study or research.

- You may not further distribute the material or use it for any profit-making activity or commercial gain

- You may freely distribute the URL identifying the publication in the public portal 


\section{Novel high-speed camera analysis of transverse mode instabilities in rod fiber amplifiers}

Christensen, Simon, Johansen, Mette, Michieletto, Mattia, Triches, Marco, Huot, Laurent, et al.

Simon L. Christensen, Mette M. Johansen, Mattia Michieletto, Marco Triches, Laurent Huot, Martin D. Maack, Jesper Lægsgaard, "Novel high-speed camera analysis of transverse mode instabilities in rod fiber amplifiers," Proc. SPIE 11260, Fiber Lasers XVII: Technology and Systems, 1126017 (21 February 2020); doi: 10.1117/12.2542673 


\title{
Novel high-speed camera analysis of transverse mode instabilities in rod fiber amplifiers
}

\author{
Simon L. Christensen ${ }^{\mathrm{a}, \mathrm{b}}$, Mette M. Johansen ${ }^{\mathrm{b}}$, Mattia Michieletto ${ }^{\mathrm{b}}$, Marco Triches ${ }^{\mathrm{b}}$, Laurent \\ Hout $^{\mathrm{b}}$, Martin D. Maack ${ }^{\mathrm{b}}$, and Jesper Lægsgaard ${ }^{\mathrm{a}}$ \\ ${ }^{a}$ DTU Fotonik, Technical University of Denmark, Ørsteds Plads, DK-2800 Kgs. Lyngby, \\ Denmark \\ ${ }^{\mathrm{b} N K T}$ Photonics A/S, Blokken 84, DK-3460 Birkerød, Denmark
}

\begin{abstract}
In this work a novel accurate method of measuring beam fluctuations is presented and applied to analyze transverse mode instabilities (TMI). The new measurement, ST-measurement, uses Fourier analysis on data from a high-speed camera to achieve raw spatial information about beam fluctuations. TMI in a $65 \mu \mathrm{m}$ modefield-diameter aeroGain-ROD-PM85 fiber is investigated using both the ST- and standard photo detector measurement. A comparison of the two measurements shows the quantitative and qualitative superiority of the new ST-measurement due to the spatial information. Numerical simulations are carried out to support the interpretation of the data.
\end{abstract}

Keywords: Transverse mode instability, Beam fluctuation measurement, High power, Thermal Rayleigh scattering, High-speed camera, Large-mode-area fiber

\section{INTRODUCTION}

For nearly a decade, the limiting factor for increasing the average power of large-mode-area (LMA) single-mode (SM) fiber amplifiers has been TMI. Many experiments and simulations have since the first observations ${ }^{1}$ been performed to describe the nature of these instabilities.

Shortly after observing TMI with a standard slow camera (25 Hz frame rate), experiments measuring TMI with a high-speed camera were performed ${ }^{2,3}$ to fully describe the beam fluctuations spatially and temporally. These experiments showed fluctuation time scales of ms and concluded dynamic energy transfer between two or more modes. The experiments also indicated three stages of TMI, the stable region, the transition region, and the chaotic region. High-speed cameras are expensive and consequently a cheaper measurement utilizing a photo detector (PD-measurement) has since then been the standard method of measuring TMI.

It is generally accepted that TMI is caused by a non-linear thermal effect. ${ }^{4,5}$ The amplification process introduces a heat load in the fiber which increases the temperature. The refractive index of the fiber is related to the temperature through the thermo-optic coefficient allowing for non-linear interaction of the signal light with itself. Many models ${ }^{6-10}$ with different assumptions and simplifications have been developed to describe this interaction and ultimately to find solutions for suppressing TMI. The problem is not trivial to solve and variables that are difficult to estimate for the amplifier systems, such as input higher-order-mode (HOM) coupling and noise, contribute to fitting of the many different models. While some ways of increasing the TMI threshold have already been proposed and shown ${ }^{11}$ other simpler solutions are needed.

Some fundamental questions regarding the amplification process and transition region of TMI still need answering even though a large amount of work has been performed both experimentally and numerically. The PD-method has been a very efficient way to find the onset of instabilities, but the elimination of the spatial dependence loses important information regarding the fluctuations. In this work we propose a novel method of measuring beam fluctuations allowing for improved understanding of TMI.

Further author information: (Send correspondence to S.L.C.)

S.L.C.: E-mail: slon@fotonik.dtu.dk

Fiber Lasers XVII: Technology and Systems, edited by Liang Dong, Proc. of SPIE Vol. 11260, 1126017 (C) 2020 SPIE · CCC code: $0277-786 X / 20 / \$ 21 \cdot$ doi: 10.1117/12.2542673 


\section{HIGH-SPEED CAMERA ANALYSIS OF TRANSVERSE MODE INSTABILITY}

In this section, the novel analysis method, spatially and temporally resolved imaging (ST-measurement), of TMI is described and investigated numerically. The novel measurement involves a high-speed camera with a capture rate faster than the time scale of the dynamics of TMI.

The previous work by Stutzki et al. ${ }^{2}$ utilizing a high-speed camera to describe TMI, analyzed the beam dynamics using an algorithm to extract the mode content at each picture of the movie. The algorithm was based on minimizing the residuals by coherently combining three calculated modes. This analysis finds the general picture in the region where a large amount of energy is transferred between modes but struggles for small fluctuations. To investigate the transition region of TMI a different analysis method is needed. The STmeasurement we propose is quite simply a Fourier Transform of the movie and consequently, no assumptions regarding the modes or their dynamics are needed. The analysis proposed is easy to perform and can be applied on all movies of beam fluctuations. The ST-measurement allows for raw information of small beam fluctuations and the resulting data can be difficult to analyze intuitively.

moves the problem of describing the beam fluctuations from the analysis to the interpretation, which will be shown can be ambiguous for some fluctuations.

When saving a movie of the beam, the intensity as a function of space and time is captured. Fourier transforming the movie with respect to time results in a complex function depending on space and frequency,

$$
\tilde{I}(x, y, \nu)=\int_{-\infty}^{\infty} I(x, y, t) \mathrm{e}^{-2 \pi \nu t} \mathrm{~d} t
$$

where $I(x, y, t)$ is captured by the movie. The intensity as a function of time is captured for each pixel in the movie. A spectrum is found for each pixel by Fourier transforming, which describes the fluctuations for that specific pixel. The absolute value and angle of $\tilde{I}(x, y, \nu)$ represent the intensity and phase, respectively. This spatial distribution of the intensity and phase can be shown, gaining significant information about the beam dynamics.

The ST-method proposed resembles in many ways that of a reduced $\mathrm{S}^{2}$-measurement, ${ }^{12}$ hence the name ST. $\mathrm{S}^{2}$ is short for spatially and spectrally resolved imaging and may be used to determine fraction of power in different modes by assuming mode beating. Calculating the fractions of power in different modes for the beam fluctuations of TMI is not possible due to the dynamic energy transfer between modes. The data generated from Fourier transforming the movie can be viewed as raw data of the fluctuations and should be treated as such. In this work, we make no assumptions regarding the dynamics of TMI to nuance the interpretation. Fundamentally different beam fluctuations are difficult to differentiate and examples of are given below.

The ST-measurement also achieves non-averaged information regarding the strength of fluctuations as long as the full beam is properly defined by the pixels. In comparison, the PD-measurement suffers from a major difficulty when dealing with measuring instabilities. The placement and size of the pinhole strongly affect the measured fluctuations. ${ }^{3}$ The PD-measurement will always weight different fluctuations differently, even with a proper placement and size of the pinhole. A consequence of this is unreliable comparison of the magnitude of peaks in the TMI spectrum. With the high-speed camera the total strength of fluctuation for a given frequency can be found by integrated the Fourier transformed intensity over all pixels. This operation achieves a single spectrum for the full instability in the beam and does not suffer averaging.

\section{Numerical implementation of mode beating and beam movement}

In the following section, two different beam fluctuations are simulated to show the resulting ST-measurement. In the simulations of the beam fluctuations a step-index-fiber (SIF) with a normalized frequency of 3 is used to create $\mathrm{LP}_{01}$ and $\mathrm{LP}_{11}$ modes.

The first simulated beam fluctuation is a simple beating in time between the $\mathrm{LP}_{01}$ and $\mathrm{LP}_{11}$ modes. In figure 1, the resulting ST-analysis is shown for a beating frequency of $300 \mathrm{~Hz}$ and $98 \%$ power in the $\mathrm{LP}_{01}$-mode and $2 \%$ power in the $\mathrm{LP}_{11}$-mode. A chaotic noise term has been added to the simulated movie to achieve a dynamic range similar to that of the high speed camera used for experiments. The figure clearly shows a single 
peak at the beating frequency which is expected for mode beating. The DC component will in this example be the sum of the intensities of the two modes, while the peak at $300 \mathrm{~Hz}$ will be the product of the two electrical fields,

$$
I(x, y, t)=a \psi_{01}^{2}+b \psi_{11}^{2}+2 \sqrt{a b} \psi_{01} \psi_{11} \cos \left(\omega_{\text {beat }} t+\phi\right)
$$

where $\psi_{01}$ and $\psi_{11}$ are the real electrical fields depending on $x$ and $y$ of the the $\mathrm{LP}_{01}$-mode and LP 11 -mode, respectively, $a$ and $b$ are the linear proportional to the powers in each mode, $\omega_{b e a t}$ is the beating angular frequency, and $\phi$ is a phase difference. The DC component looks like an $\mathrm{LP}_{01}$-mode since the fraction of power in the $\mathrm{LP}_{11}$-mode is small compared to $\mathrm{LP}_{01}$. The spatial shape at the $\mathrm{AC}$ peak does not depend on the relative fraction of power between the two modes. For a pure beating dynamic, the fractions of power in each mode could be found using the $\mathrm{S}^{2}$-analysis.

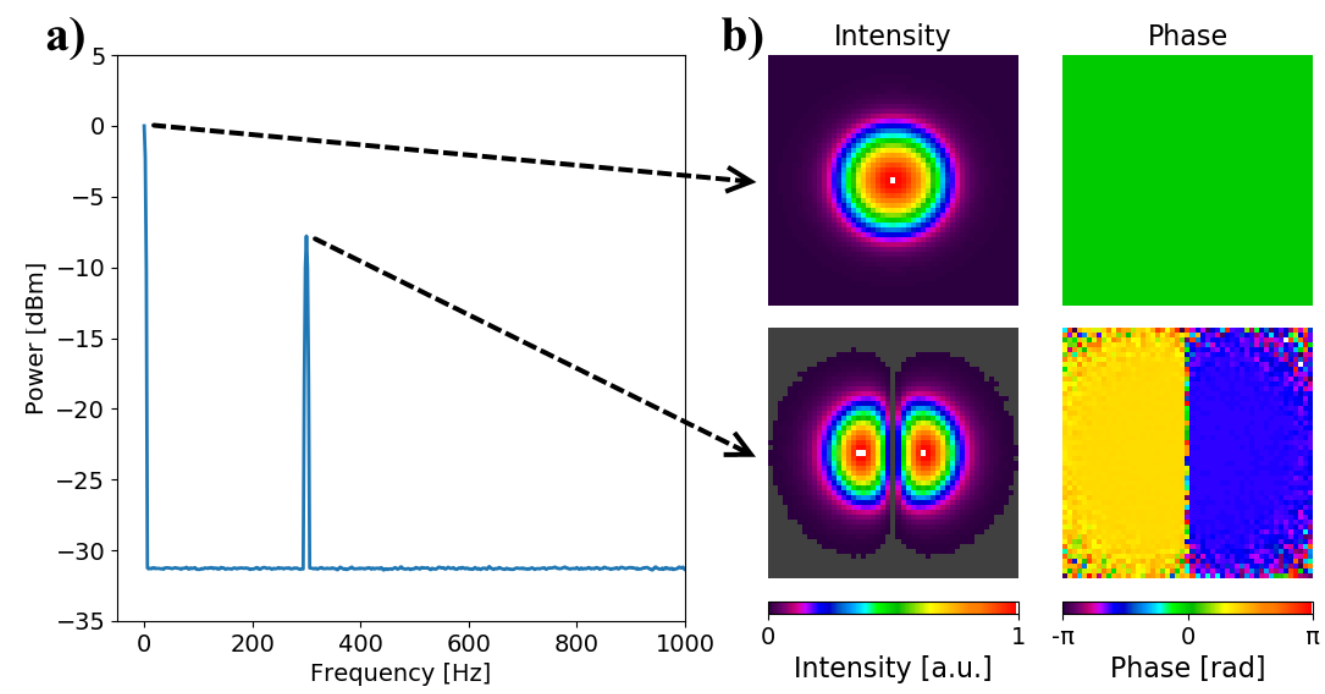

Figure 1: a) The integrated spectrum for a beat frequency of $300 \mathrm{~Hz}$ and $98 \%$ power in the $\mathrm{LP}_{01}$ and $2 \%$ power in the $\mathrm{LP}_{11}$. b) Spatial intensity and phase of the Fourier transform for the DC and $300 \mathrm{~Hz}$ peak.

Different effects may appear similar when considering the spatial intensity and phase form the ST-measurement. This is illustrated by simulating an $\mathrm{LP}_{01}$ moving in time. In this simulation, the $\mathrm{LP}_{01}$ follows a sinusoidal displacement with a given frequency and amplitude. The movie resulting from this effect is described by

$$
I(x, y, t)=a\left|\psi_{01}(x+\Delta x, y)\right|^{2}, \Delta x=A \sin \left(\omega_{\text {move }} t+\phi\right)
$$

where $A$ and $\omega_{\text {move }}$ is the amplitude and moving angular frequency of the displacement, respectively, and $\phi$ is a phase difference. In figure 2 , the resulting ST-measurement is shown for a displacement frequency of $300 \mathrm{~Hz}$ and an amplitude of $1 / 10$ of the core radius. A noise term similar to the beating noise has been applied. The ST-measurement of the moving beam shows similar behaviour as the pure mode beating in figure 1 for the DC peak and the largest AC peak. The spatial phase plot of the peak at $300 \mathrm{~Hz}$ also resembles the phase plot of the pure beating since the two lobes have a $\pi$-shift in phase. However a Taylor expansion of the moving beam profile reveals that the spatial distribution originates from the $\mathrm{x}$-derivative of the $\mathrm{LP}_{01}$ intensity. The moving beam also has higher harmonics of the movement which can be seen at $600 \mathrm{~Hz}$ and $900 \mathrm{~Hz}$ and further Taylor expansion reveals that these spatial distributions are mainly determined by the second and third-order x-derivatives of the $\mathrm{LP}_{01}$ intensity. Consequently the distribution at $600 \mathrm{~Hz}$ does not show mode shapes and can be used to determine the underlying beam fluctuation as a beam movement. The problem of distinguishing between the two effects arises when the second harmonic of the moving beam drops below the noise floor, which will happen for a sufficiently small amplitude of the movement. In this regime it will be very difficult to determine the cause 


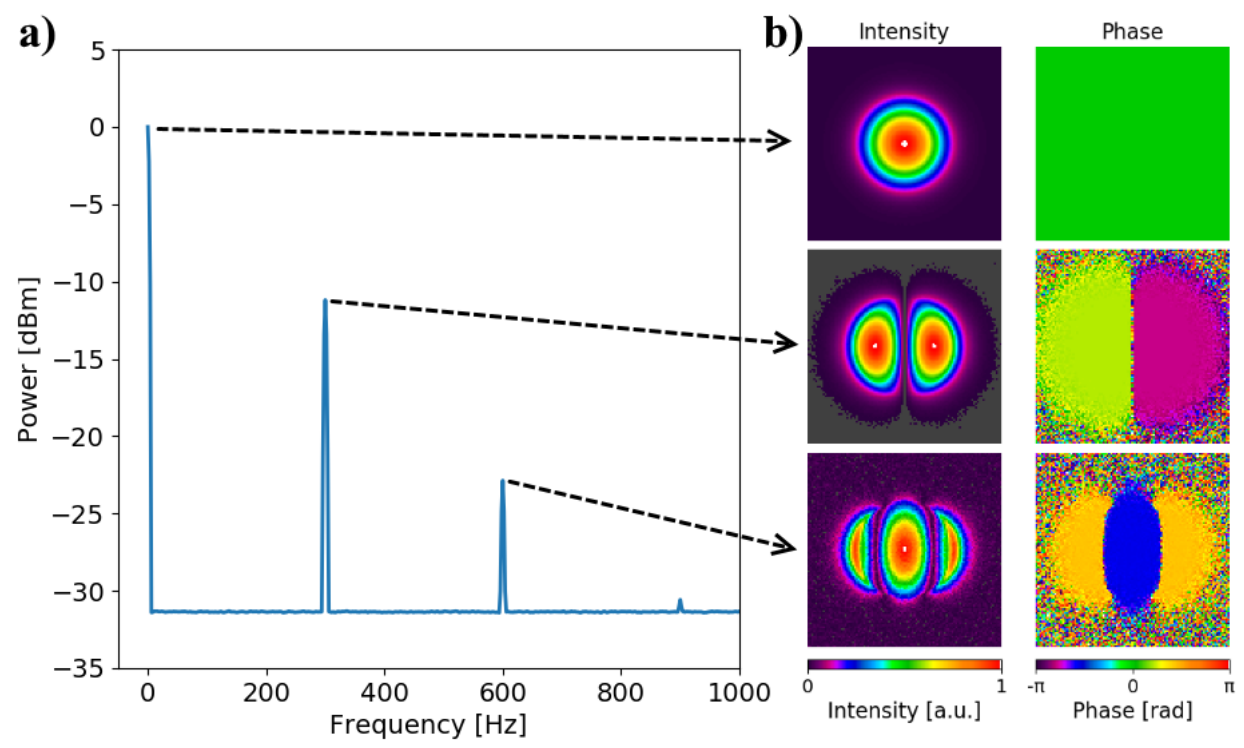

Figure 2: a) The integrated spectrum for a beam moving with a frequency of $300 \mathrm{~Hz}$ and an amplitude of $1 / 10$ of the fiber core radius. b) Spatial intensity and phase of the Fourier transform for the DC, $300 \mathrm{~Hz}$, and 600 $\mathrm{Hz}$ peak.

of the fluctuation without having some other information of the system , and therefore careful interpretation of the results is needed. Also, the picture becomes increasingly more complex when dealing with dynamic transfer of energy between multiple modes, which is out of scope for this work.

\section{MEASUREMENTS OF TMI}

The ST-measurement is applied to experimental data of TMI. Different measurements are performed to showcase the enormous amount of information the ST-analysis provides.

In this work all measurements are performed on an $80 \mathrm{~cm}$ aeroGAIN-ROD-PM85 fiber with a $65 \mu \mathrm{m}$ MFD from NKT Photonics. ${ }^{13}$ This fiber mainly has TMI gain from 200 to $800 \mathrm{~Hz}$ depending on the strength of the TMI and a Basler camera (model acA640-750um ${ }^{14}$ ) is used to perform the ST-measurement. For a reduced scanning area the camera can achieve 4000 frames per second which is enough to capture the important dynamics of the TMI fluctuations. A sketch of the setup for measuring beam dynamics at TMI powers can be seen in figure 3.

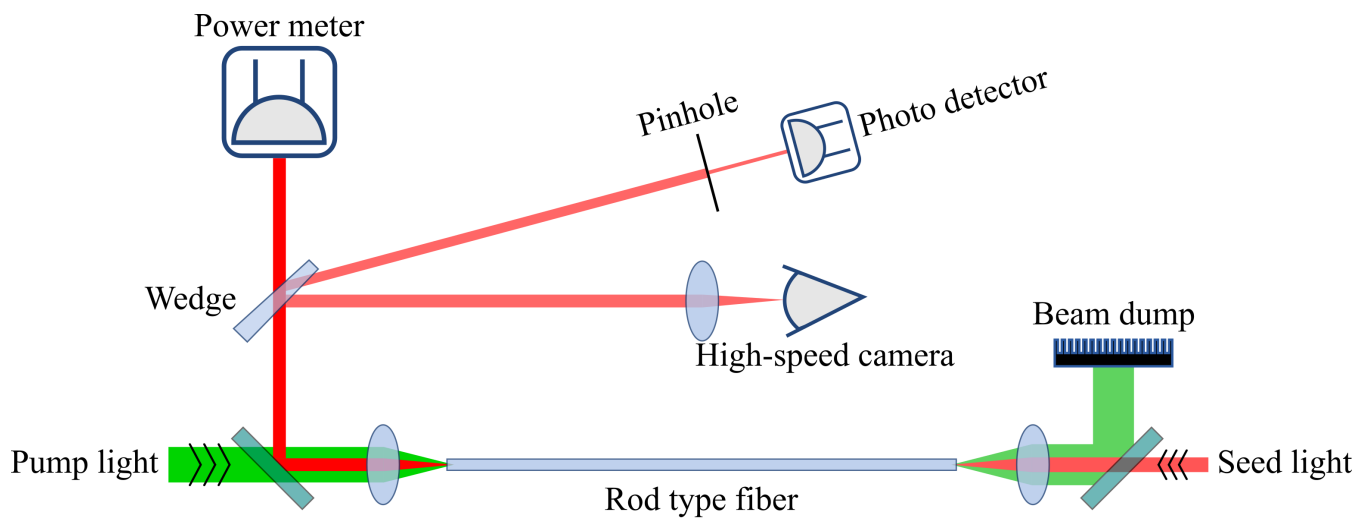

Figure 3: Sketch of the fiber amplifier setup. 


\section{ST-measurements of TMI with a high-speed camera}

A pristine rod is backwards pumped by a laser diode to achieve amplification of a seed laser. The input seed light wavelength is $1030 \mathrm{~nm}$ and an input seed power of $15 \mathrm{~W}$ was used for all measurements presented. The measurements consist of data from both the fast camera and photo detector at different signal powers. This section presents the ST-measurements for two signal powers.

The ST-measurement for a signal power below the TMI threshold is shown in figure 4. The noise spectrum is generally flat above $150 \mathrm{~Hz}$ while some fluctuations are observed for smaller frequencies. The figure shows four different spatial intensity distributions and phase plots at 0, 8, 28 and $1284 \mathrm{~Hz}$. The DC does not show any sign of deformation, which is expected for signal powers below the TMI threshold. The two peaks at 8 and $28 \mathrm{~Hz}$ have quite interesting spatial distributions and do not originate from noise. These fluctuations are very slow compared to the expected TMI frequencies of the fiber. The origin of these fluctuations is most likely not beating with higher order modes, but a consequence of beam movement. The most probable explanation is air turbulence around the output lens due heating of this component causing beam translations at the high speed camera. The fluctuation at $8 \mathrm{~Hz}$ could be a linear movement of the beam while the fluctuation at 28 $\mathrm{Hz}$ is a circular movement. These low frequency fluctuations persisted for some minutes when turning off the pump after high-power amplification. For the amplitude of these movements the higher order components will be below the noise floor and consequently not detectable. The small peak at $1284 \mathrm{~Hz}$ is an error caused by the camera detection which is seen by the strange line in the spatial intensity and phase.

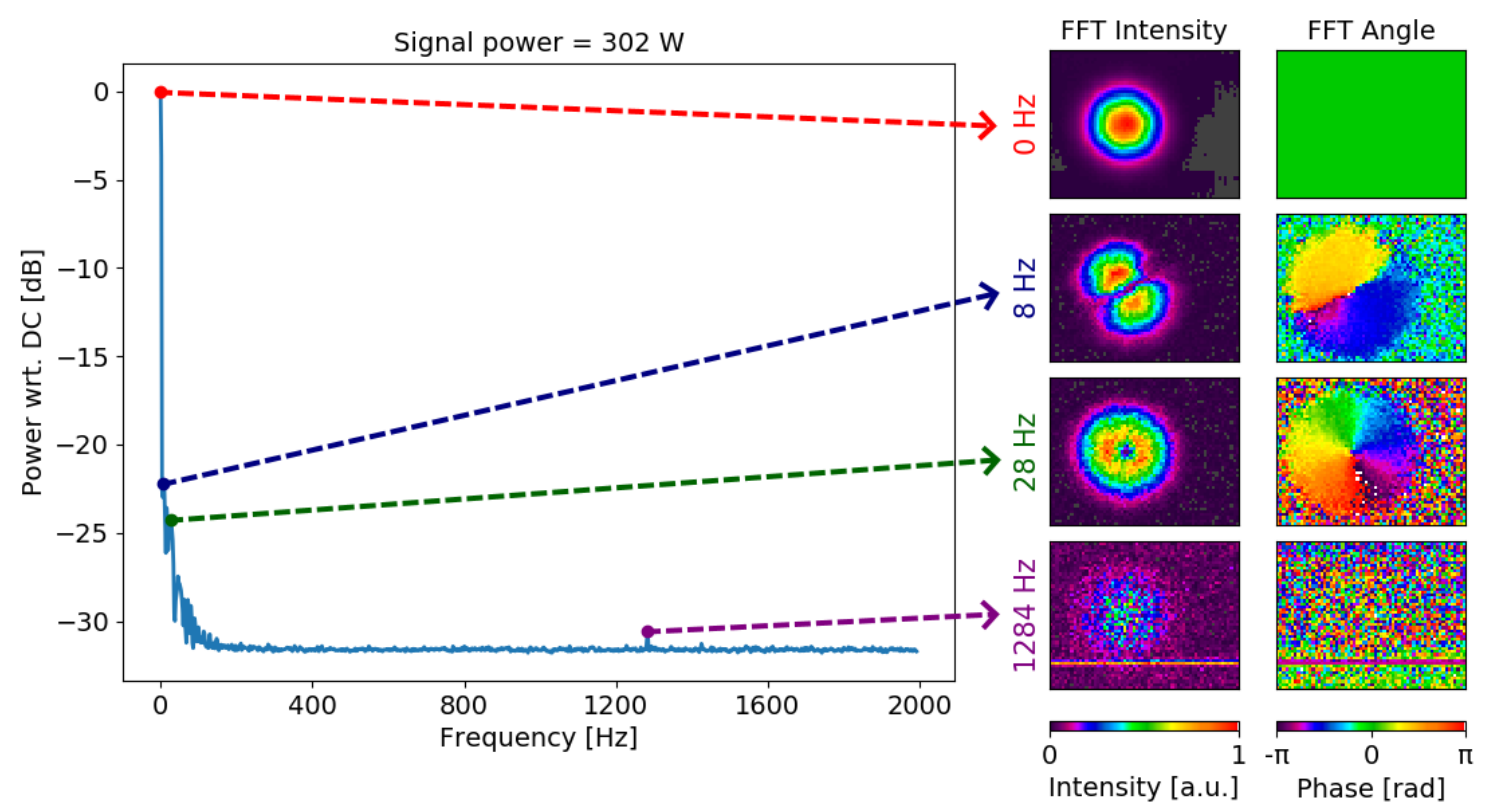

Figure 4: ST-measurement for a rod fiber for a signal power of $302 \mathrm{~W}$.

The ST-measurement becomes significantly more interesting when crossing the TMI threshold. A part of the ST-measurement for a signal power above the TMI threshold can be seen in figure 5. This power level is inside the TMI transition region and the spatial intensity of the DC peak (not shown) is still Gaussian. The spatial intensity and phase of four peaks at 10,301,399, and $601 \mathrm{~Hz}$ are shown, and the low frequency peak at $10 \mathrm{~Hz}$ is still believed to be due to air turbulence.

The three other peaks shown in the figure all look very similar with respect to distribution and orientation. They all fall inside the expected TMI gain band and therefore assumed to originate from the same type of beam fluctuation caused by interaction between the $\mathrm{LP}_{01}$ and $\mathrm{LP}_{11}$ modes. The similar rotation for all the TMI fluctuations suggests a preferred orientation of the $\mathrm{LP}_{11}$ in the fiber. For all other peaks with a significant magnitude inside the TMI gain band the spatial distributions are similar to the distributions shown at 301, 


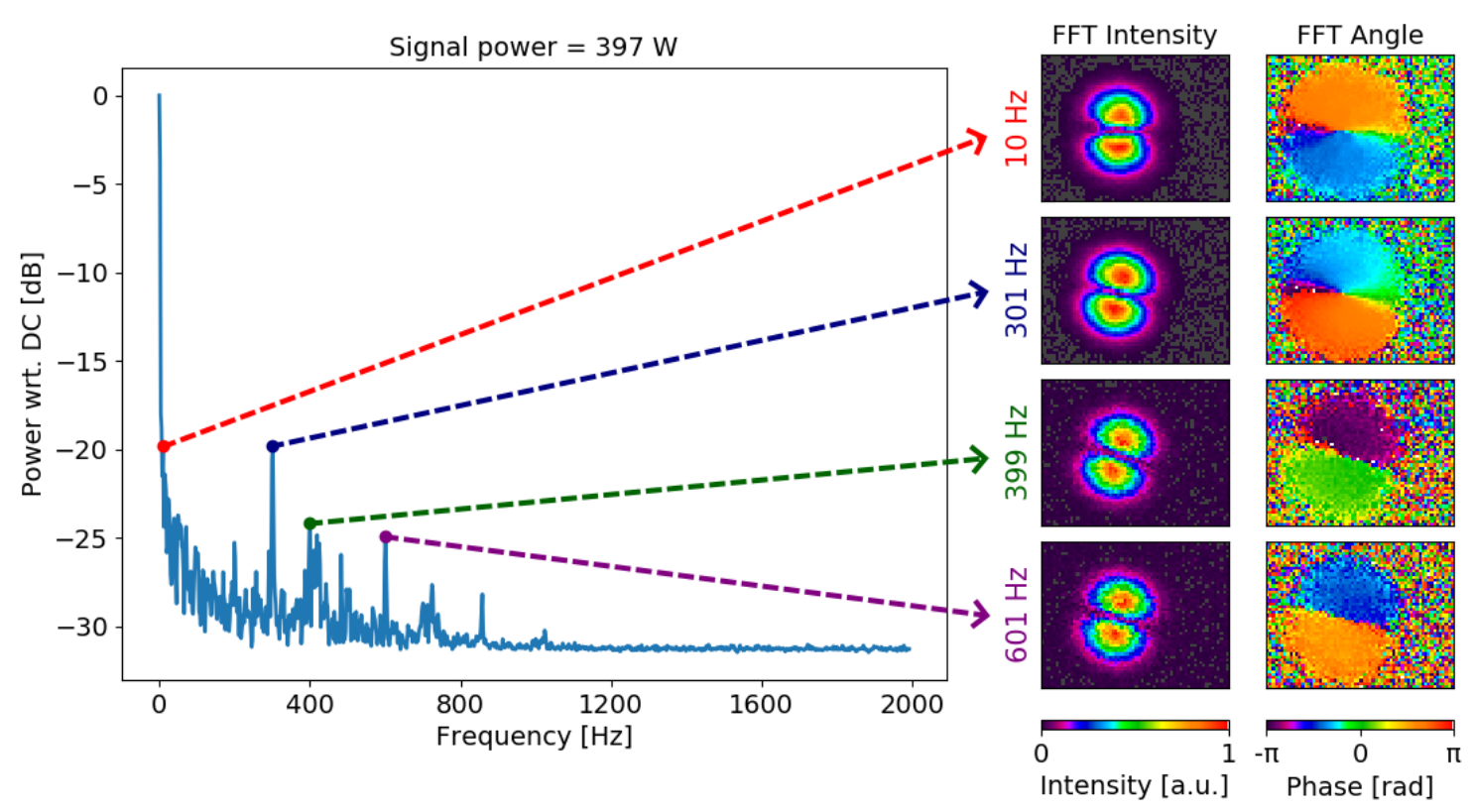

Figure 5: ST-measurement for a rod fiber for a signal power of $397 \mathrm{~W}$.

399, and $601 \mathrm{~Hz}$. Interaction with other HOM are not observed for this signal power or other signal powers investigated.

\section{Comparison between ST and PD-method}

The fluctuation spectra have been normalized to have a DC peak at 0 dB. Figure 6 shows the fluctuation spectrum for both the high-speed camera and the photo detector at the signal power level of figure 5 . The PD-measurement underestimates the magnitude of all peaks but not with the same factor. This is a problem for the absolute value of the fluctuation and the relative magnitude between peaks.

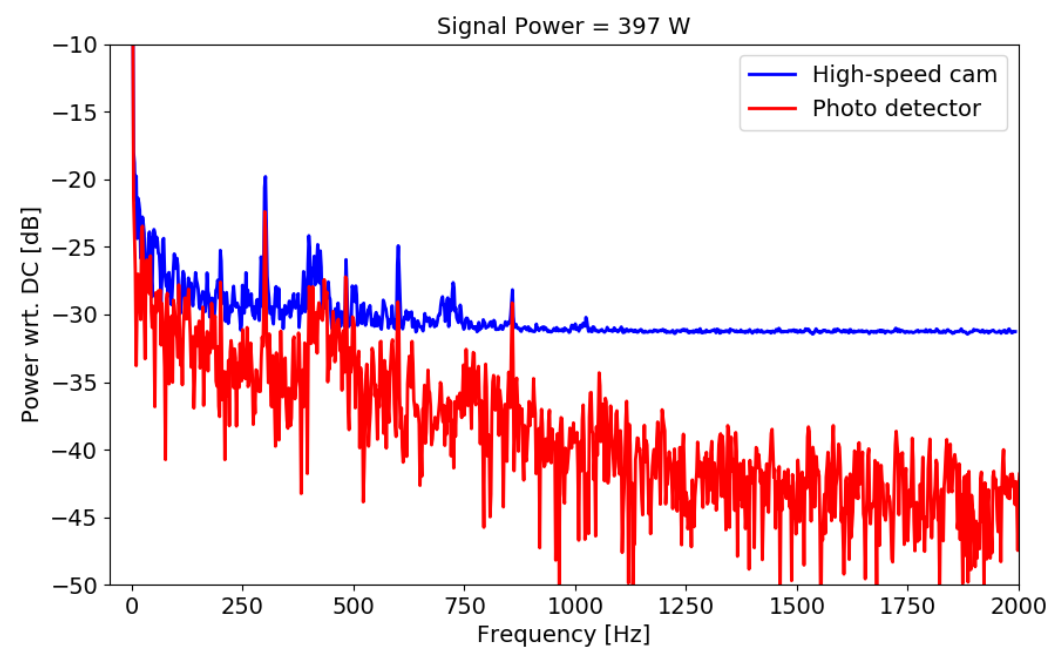

Figure 6: Spectrum of the signal fluctuations for both the ST and PD-method for a signal power of $397 \mathrm{~W}$. 
The comparison shows that the noise level is much lower for the PD, which is expected due to the larger dynamic range. This means that the PD will be able to detect smaller fluctuations of the beam. The acquisition rate of the $\mathrm{PD}$ is also higher, enabling detection of faster fluctuations. Considering only these two aspects the $\mathrm{PD}$ is superior to a high-speed camera. However the lower dynamic range and speed will only be a problem for the ST-measurement, when the fluctuations are faster than the acquisition rate of the camera or weaker than the noise of the camera. The superiority of the ST-measurement is undebatable due to the massive amount of information regarding the spatial properties of each fluctuation. As mentioned previously, the PD will in most cases misrepresent the magnitude of fluctuations depending on spatial intensities of the fluctuations due to pinhole position and size.

The integrated power above $150 \mathrm{~Hz}$ in the fluctuation spectrum is used to evaluate the TMI level. This figure of merit does not fully describe the fluctuations but will approximate the TMI strength. A cut-off frequency of $150 \mathrm{~Hz}$ is chosen to filter out the slow movement fluctuations below this frequency. In figure 7 the fraction of power in TMI is shown as a function of signal power for both the ST and PD-method. When calculating the fraction of power above $150 \mathrm{~Hz}$ only magnitudes above the noise level are used i.e. points below the noise level are set as zero. This underestimates the true fraction of power for weaker fluctuations but also allow for better information regarding the TMI gain.

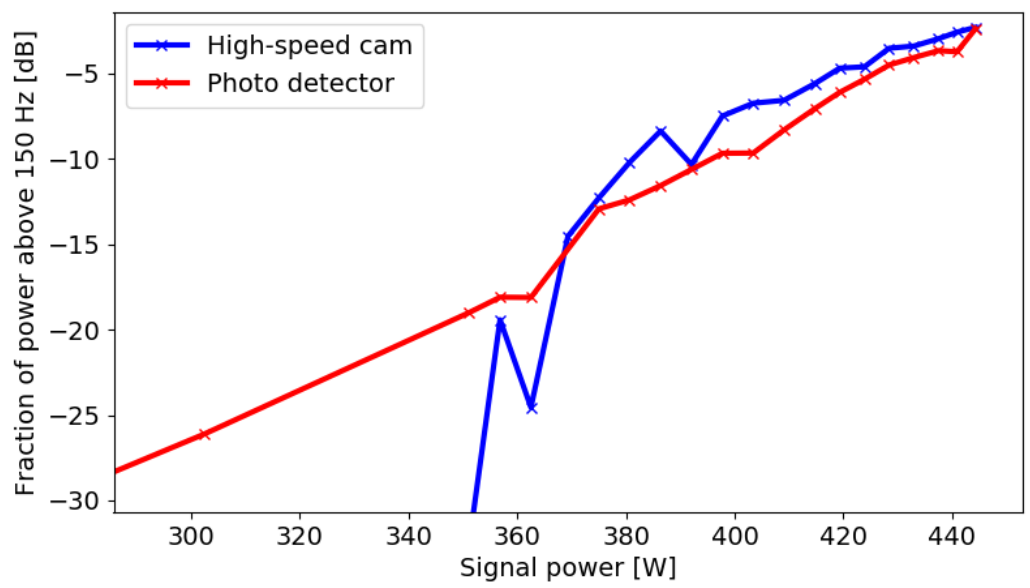

Figure 7: Integrated power above $150 \mathrm{~Hz}$ for both the ST and PD-method.

The fraction of power in fluctuations as a function of signal power clearly shows the superiority of the PDmethod for weaker fluctuations due the larger dynamic range. For these signal powers the ST-method does not yield results of value since the fluctuations are below the noise level of the camera. The fraction power in the TMI becomes significantly large for signal powers around $380 \mathrm{~W}$ and above, and the ST-measurement predicts larger fractions of power in the fluctuations. This measurement shows that the PD-method generally captures the gain of TMI quite well even though the absolute value is underestimated. The result of the PD shows that fraction of power in the fluctuations follows an exponential growth well.

The preferred method for measuring TMI will depend on the experiment. For experiments only interested in the threshold of TMI, the PD-method is sufficient. While experiments dealing with the spectral shape of the TMI gain or beam fluctuation dynamics requires the ST-method.

\section{CONCLUSION}

We have shown a novel method of measuring beam fluctuations using a cheap high-speed camera and applied this ST-measurement to measure beam fluctuations caused by TMI for a aeroGAIN-ROD-PM85 single-mode largemode-area fiber amplifier. The results of the ST-method allows for raw spatial information regarding fluctuations 
of the beam and accurately describes the magnitude of fluctuation for different frequencies. Compared to the standard photo detector method of measuring TMI the ST-method allows for more quantitative results by adding spatial information. The comparison between the ST and PD-method also showed limitations of the ST-measurement with respect to camera acquisition speed and dynamical range.

Numerical results showed that fundamentally different beam fluctuations can look similar and careful interpretation of the data is needed. Even though the ST-method has its limitations, it is expected that further experiments will lead to better description and understanding of TMI and consequently better solutions for suppressing this limitation in output power.

\section{REFERENCES}

[1] Eidam, T., Wirth, C., Jauregui, C., Stutzki, F., Jansen, F., Otto, H.-J., Schmidt, O., Schreiber, T., Limpert, J., and Tünnermann, A., "Experimental observations of the threshold-like onset of mode instabilities in high power fiber amplifiers," Opt. Express 19, 13218-13224 (Jul 2011).

[2] Stutzki, F., Otto, H.-J., Jansen, F., Gaida, C., Jauregui, C., Limpert, J., and Tünnermann, A., "Highspeed modal decomposition of mode instabilities in high-power fiber lasers," Opt. Lett. 36, 4572-4574 (Dec 2011).

[3] Otto, H.-J., Stutzki, F., Jansen, F., Eidam, T., Jauregui, C., Limpert, J., and Tünnermann, A., "Temporal dynamics of mode instabilities in high-power fiber lasers and amplifiers," Opt. Express 20, 15710-15722 (Jul 2012).

[4] Johansen, M. M., Laurila, M., Maack, M. D., Noordegraaf, D., Jakobsen, C., Alkeskjold, T. T., and Lægsgaard, J., "Frequency resolved transverse mode instability in rod fiber amplifiers," Opt. Express 21, 21847-21856 (Sep 2013).

[5] Otto, H.-J., Modsching, N., Jauregui, C., Limpert, J., and Tünnermann, A., "Impact of photodarkening on the mode instability threshold," Opt. Express 23, 15265-15277 (Jun 2015).

[6] Smith, A. V. and Smith, J. J., "Mode instability in high power fiber amplifiers," Opt. Express 19, 1018010192 (May 2011).

[7] Hansen, K. R., Alkeskjold, T. T., Broeng, J., and Lægsgaard, J., "Theoretical analysis of mode instability in high-power fiber amplifiers," Opt. Express 21, 1944-1971 (Jan 2013).

[8] Zervas, M. N., "Transverse mode instability analysis in fiber amplifiers," in [Fiber Lasers XIV: Technology and Systems], Robin, C. A. and Hartl, I., eds., 10083, 115 - 123, International Society for Optics and Photonics, SPIE (2017).

[9] Ward, B. G., "Modeling of transient modal instability in fiber amplifiers," Opt. Express 21, 12053-12067 (May 2013).

[10] Jauregui, C., Eidam, T., Otto, H.-J., Stutzki, F., Jansen, F., Limpert, J., and Tünnermann, A., "Temperature-induced index gratings and their impact on mode instabilities in high-power fiber laser systems," Opt. Express 20, 440-451 (Jan 2012).

[11] Jauregui, C., Stihler, C., Tünnermann, A., and Limpert, J., "Pump-modulation-induced beam stabilization in high-power fiber laser systems above the mode instability threshold," Opt. Express 26, 10691-10704 (Apr 2018).

[12] Nicholson, J. W., Yablon, A. D., Ramachandran, S., and Ghalmi, S., "Spatially and spectrally resolved imaging of modal content in large-mode-area fibers," Opt. Express 16, 7233-7243 (May 2008).

[13] https://www.nktphotonics.com/lasers-fibers/product/aerogain-rod-high-power-ytterbium-rod-fiber-gainmodules/ (Dec 2019).

[14] https://www.baslerweb.com/en/products/cameras/area-scan-cameras/ace/aca640-750um/ (Dec 2019). 